\title{
Proposta para estimar curva carga-recalque e capacidade de carga em estacas de madeira
}

\author{
Proposal to estimate load-settlement curve and load \\ capacity of timber piles
}

\begin{tabular}{|c|c|}
\hline & $\begin{array}{l}\text { Pedro Gutemberg de Alcântara Segundinho } \\
\text { Alexandre José Soares Miná } \\
\text { Antonio Alves Dias } \\
\text { Adair José Regazzi } \\
\text { Marcelo Rodrigo Carreira } \\
\text { Resumo }\end{array}$ \\
\hline $\begin{array}{r}\text { dro Gutemberg de Alcântara } \\
\text { Segundinho } \\
\text { Universidade Federal do Espírito } \\
\text { Santo } \\
\text { Jerônimo Monteiro - ES - Brasil }\end{array}$ & 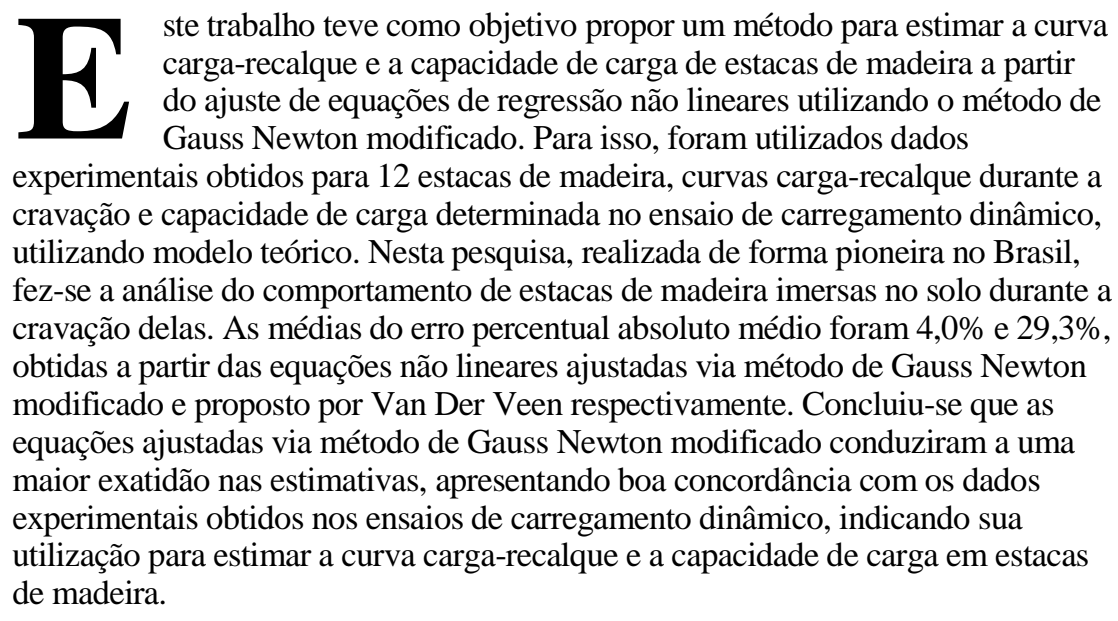 \\
\hline $\begin{array}{r}\text { Alexandre José Soares Miná } \\
\text { Universidade Federal da Paraíba } \\
\text { Bananeiras - PB - Brasil }\end{array}$ & $\begin{array}{l}\text { Palavras-chave: Estacas de madeira. Regressão não linear. Método de Gauss Newton. } \\
\text { Abstract }\end{array}$ \\
\hline $\begin{array}{l}\text { Antonio Alves Dias } \\
\text { Universidade de São Paulo } \\
\text { São Carlos - SP - Brasil }\end{array}$ & $\begin{array}{l}\text { The aim of this study was to propose a method for estimating the load-settlement } \\
\text { curve and the load capacity of timber piles, through the adjustment of nonlinear } \\
\text { regression equations, using the modified Gauss Newton method. Experimental } \\
\text { data were used for twelve timber piles, including load-settlement curves during } \\
\text { spiking, and load canacity determined at the dvnamic load testing using a }\end{array}$ \\
\hline $\begin{array}{r}\text { Adair José Regazzi } \\
\text { Universidade Federal de Viçosa } \\
\text { Viçosa - MG - Brasil }\end{array}$ & $\begin{array}{l}\text { spiking, and load capacity determined at the dynamic load testing using a } \\
\text { theoretical model. In the research (a pioneering study in Brazil) we have analyzed } \\
\text { the behavior of timber piles immersed in the ground, during spiking. The average } \\
\text { means for absolute percentage error were } 4.0 \% \text { and } 29.3 \% \text {, which were obtained } \\
\text { from adiusted }\end{array}$ \\
\hline $\begin{array}{r}\text { Marcelo Rodrigo Carreira } \\
\text { iversidade Tecnológica Federal do } \\
\text { Paraná } \\
\text { Campo Mourão - PR - Brasil }\end{array}$ & $\begin{array}{l}\text { and the method proposed by Van Der Veen, respectively. The conclusion was } \\
\text { made that equations adjusted through the modified Gauss Newton method led to } \\
\text { greater estimative accuracy, showing good concordance with the experimental } \\
\text { data obtained from dynamic load testing, which indicates its use in the estimation } \\
\text { of load-settlement curve and load capacity of timber piles. }\end{array}$ \\
\hline & Keywords: Timber piles. Nonlinear regression. Gauss Newton method. \\
\hline
\end{tabular}

SEGUNDINHO, P. G. DE A.; MINÁ, A. J. S.; DIAS, A. A.; REGAZZI, A. J.; CARREIRA, M. R. Proposta para estimar curva 29 carga-recalque e capacidade de carga em estacas de madeira. Ambiente Construído, Porto Alegre, v. 14, n. 1, p. 29-40, jan./mar. 2014.

ISSN 1678-8621 Associação Nacional de Tecnologia do Ambiente Construído. 


\section{Introdução}

As estacas de madeira são adequadas para fundações de vários tipos de estruturas, pois apresentam muitas vantagens: baixo custo, alta resistência mecânica para solicitações estáticas e dinâmicas, baixo peso, facilidade de transporte e de processamento (FREEDMAN et al., 2002). Além disso, também possuem grande durabilidade, citando como exemplos a Old London Bridge, construída em 1176, a Pont Notre-Dame, construída em 1412, em Paris, e pequenas pontes na América do Norte, construídas desde meados do século XVIII (FREEDMAN et al., 2002; AMERICAN..., 2002), mesmo que, antes de 1900, todas as estacas fossem de madeira sem tratamento (FREEDMAN et al., 2002). Tschebotarioff (1978) cita um caso histórico ocorrido durante a reconstrução do campanário da Igreja de São Marcos, em Veneza, em 1902, quando se verificou que as estacas de madeira, após mil anos de serviço, estavam em tão boas condições que foram mantidas no local para a fundação da nova torre.

As estacas de madeira apresentam durabilidade quase que ilimitada quando trabalham completamente abaixo do lençol freático; ou seja, sob condições ideais, confinadas abaixo do nível da água, elas são virtualmente imunes ao ataque biológico e têm vida útil quase indefinida (FREEDMAN et al., 2002). Acima do nível da água, devem ser tratadas com preservantes adequados, aplicados sob pressão, em autoclaves, para evitar a rápida deterioração, sendo esta a principal desvantagem quando submetida à variação de umidade, que pode ser eficaz durante pelo menos 40 anos (PECK; HANSON; THORNBURN, 1974).

Segundo Décourt (1996), uma estaca prismática submetida a um carregamento vertical resistirá a essa solicitação, em parte, pela resistência ao cisalhamento gerada ao longo de seu fuste e, em parte, pelas tensões normais geradas no nível de sua base. As estacas de madeira possuem conicidade natural, o que proporciona aumento em sua capacidade de carga.

A American Society for Testing and Materials ASTM D-25 (AMERICAN..., 1999) define as características físicas (dimensões mínimas, comprimentos disponíveis) e as qualidades necessárias (taxa mínima de anéis de crescimento e porcentagem de madeira de verão, retidão, retirada da casca, limitação de conicidade, limitação de fibras retorcidas, limitação de nós e outros defeitos) de estacas roliças de madeira, tratadas ou não, para ser usadas em fundações.
As estacas de madeira são projetadas ou classificadas, principalmente, para situações de carregamento de compressão centrada, partindo do princípio de que a superestrutura proporciona rigidez suficiente para prevenir a excentricidades nas estacas segundo a norma da American Associated of State Highway Officials (AMERICAN..., 2007). No entanto, segundo Borello et al. (2010), a suposição de compressão centrada em estacas de madeira não é obrigatória para superestrutura que consista em vãos simplesmente apoiados, mas o efeito da interação de compressão, flexão, sobre estacas de madeira em pontes deve ser observado durante a concepção da estrutura, em especial no caso das superestruturas simplesmente apoiadas em que o carregamento de um intervalo pode levar a carga excêntrica em um grupo de várias estacas.

A superestrutura das pontes de madeira de pequeno vão apresenta preço competitivo quando comparada com a superestrutura construída com outros materiais estruturais, como o concreto e o aço (OKIMOTO; CALIL JÚNIOR, 1998), o que indica uso adequado para subestrutura em estacas de madeira.

O conhecimento, mesmo que aproximado, da curva carga-recalque de uma fundação é fundamental, tanto para a correta interpretação dos dados de provas de carga, quanto para o correto dimensionamento (DÉCOURT, 1996). De acordo com Alonso (1991), a previsão da curva cargarecalque pode ser feita por meio de uma equação matemática desde que se calcule para vários níveis de carregamento $\left(Q_{i}\right)$ o correspondente recalque $\left(W_{i}\right)$. Segundo a Norma Brasileira Regulamentadora - NBR 6122 da Associação Brasileira de Normas Técnicas - (ABNT, 2010), a capacidade de carga do elemento de prova deve ser considerada, e definida quando ocorrer ruptura nítida; porém, o carregamento da estaca de prova pode não indicar uma carga de ruptura clara, e isso ocorre quando não se pretende levar a fundação à ruptura ou quando a estaca tem capacidade de resistir a uma carga maior do que aquela que se pode aplicar na prova (por limitação de reação), ou, ainda, quando a estaca é carregada até apresentar um recalque considerável.

Os métodos estáticos teóricos são fundamentais para estabelecer a influência relativa dos fatores envolvidos na determinação da capacidade de carga de estacas, mas a utilização prática desses métodos é restrita devido à dificuldade de obtenção dos parâmetros do solo necessário (DÉCOURT, 1996). Velloso e Lopes (2002) afirmam que os critérios utilizados para 
determinação da capacidade de carga podem ser agrupados em quatro categorias: (i) critérios baseados num valor absoluto de recalque; (ii) critérios baseados na aplicação de uma regra geométrica à curva; (iii) critérios que buscam uma assíntota vertical; e (iv) critérios que caracterizam a ruptura devido ao encurtamento elástico da estaca obtido por procedimento gráfico indicando o repique e a nega (CHELLIS, 1961). O critério adotado pela NBR 6122 (ABNT, 2010) se insere na quarta categoria, aplicado quando o ensaio não é feito até a ruptura. A extrapolação da curva carga-recalque é baseada numa função matemática ajustada aos dados registrados e apresenta uma assíntota correspondente à carga de ruptura, conforme critérios da categoria três.

No Brasil, a função matemática mais utilizada para a extrapolação da curva carga-recalque é aquela proposta por Van Der Veen (1953), que, de acordo com Aoki (1997), é um método válido quando o valor da carga de ruptura extrapolada for menor que o dobro da carga máxima aplicada. A metodologia para determinação da curva compreende a estimativa da capacidade de carga e o cálculo do recalque correspondente à carga de trabalho, devendo esta ser menor que a metade daquela. Aplicando-se os valores da capacidade de carga, da carga de trabalho e de seu recalque correspondente, pode-se determinar o valor do coeficiente $\delta_{a}$, que depende das características da estaca e da natureza do solo, e, consequentemente, traçar a curva carga-recalque teórica por meio da Equação 1, conforme Van Der Veen (1953).

$Q_{i, v}=Q_{u, a}\left(1-e^{-\delta_{a} W_{i}}\right), \quad i=1,2, \ldots, n$,

Eq. 1

Em que:

$Q_{i, v}$ : carga correspondente ao recalque $W_{i}(\mathrm{kN})$;

$W_{i}$ : recalque correspondente à carga $Q_{i, v}(\mathrm{~mm}) ;$

$Q_{u, a}$ : capacidade de carga obtida conforme Aoki e Velloso (1975) (kN); e

$\delta_{a}$ : coeficiente que define a forma da curva,

determinado a partir do recalque total $\left(W^{*}\right)$ correspondente à carga de $250 \mathrm{kN}$, segundo Chellis (1961), $\left(\mathrm{mm}^{-1}\right)$.

O objetivo deste trabalho foi, portanto, propor um método para estimar a curva carga-recalque e a capacidade de carga em estacas de madeira a partir do ajuste de equações não lineares utilizando o método de Gauss Newton modificado. O estudo foi realizado em 12 estacas de madeira durante a cravação, com a finalidade de obter as curvas carga-recalque e capacidade de carga a partir dos dados experimentais obtidos por meio de ensaio de carregamento dinâmico e do método teórico de
Van Der Veen (1953). Nesta pesquisa, realizada de forma pioneira no Brasil, fez-se a análise do comportamento de estacas de madeira imersas no solo, durante a cravação delas, a partir dos dados experimentais obtidos no ensaio de carregamento dinâmico.

\section{Estimativa da curva carga- recalque e da capacidade de carga utilizando o Método de Gauss Newton modificado}

Na primeira etapa, para cada uma das 12 estacas de madeira, obteve-se uma equação segundo o modelo teórico proposto por Van Der Veen (1953), com a finalidade de obter os valores iniciais dos parâmetros da capacidade de carga $\left(Q_{u, a}\right)$ e do coeficiente $\left(\delta_{g}\right)$, que define a forma da curva cargarecalque (Equação 1).

$\mathrm{Na}$ segunda etapa, para cada estaca, foi considerado o seguinte modelo estatístico de regressão não linear (Equação 2):

$Q_{i, g}=Q_{u, g}\left(1-e^{-\delta_{g} W_{i}}\right)+e_{i}, \quad i=1,2, \ldots, n$.

Eq. 2

Os parâmetros $Q_{u, g}$ e $\delta_{g}$ foram estimados pelo método de Gauss Newton modificado, conforme Souza (1998). Para cada uma das 12 equações ajustadas foram calculadas as estatísticas a seguir.

Coeficiente de determinação ajustado, expressão proposta por Kvalseth (1985) para modelos de regressão não linear (Equação 3):

$R_{a j .}^{2}=1-\left(\frac{n-i}{n-k}\right)\left[1-\left(1-\frac{S Q R}{S Q T \text { otal }_{\text {corrigida }}}\right)\right]$

Eq. 3

Em que:

$i$ : igual a 1 se o modelo inclui o intercepto;

$i$ : igual a 0 se o modelo não inclui o intercepto;

$k$ : número total de parâmetros;

$S Q R$ : soma de quadrados do resíduo; e

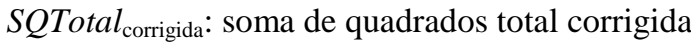
pela média.

Assim, para o modelo em questão, tem-se (Equação 4):

$R_{a j .}^{2}=1-\left(\frac{n}{n-2}\right)\left[1-\left(1-\frac{S Q R}{S Q \text { total }_{\text {corrigida }}}\right)\right]$

Eq. 4

Intervalo de confiança para os parâmetros $Q_{u, g}$ e $\delta_{g}$ no nível de confiança de $95 \%$ (Equações 5 e 6 ):

$I C\left(Q_{u, g}\right)_{95 \%}: \hat{Q}_{u, g} \pm t_{\left(\frac{\alpha}{2} ; n-k\right)} \cdot \sqrt{\hat{V}\left(\hat{Q}_{u, g}\right)}$

Eq. 5 
$\operatorname{IC}\left(\delta_{g}\right)_{95 \%}: \hat{\delta}_{g} \pm t_{\left(\frac{\alpha}{2} ; n-k\right)} \cdot \sqrt{\hat{V}\left(\hat{\delta}_{g}\right)}$

Eq. 6

Para comparar os valores $Q_{i, v}$ (carga obtida de acordo com o que Van Der Veen (1953) propõe) e os valores $\hat{Q}_{i, g}$ (carga predita pelas equações de regressão não lineares ajustadas pelo método de Gauss Newton modificado) com os valores obtidos experimentalmente $Q_{i, \text { exp }}$, foram utilizados as estatísticas a seguir.

Erro Absoluto Médio (EAM) (Equação 7 e 8):

$$
\begin{aligned}
& E A M=\frac{1}{n} \sum_{i=1}^{n}\left|Q_{i, \exp }-Q_{i, v}\right| \\
& E A M=\frac{1}{n} \sum_{i=1}^{n}\left|Q_{i, \exp }-\hat{Q}_{i, g}\right|
\end{aligned}
$$

Erro Percentual Absoluto Médio (EPAM) (Equação 9):

$$
E P A M=\frac{1}{n} \sum_{i=1}^{n}\left|E P_{i}\right|
$$

$\mathrm{Na}$ Equação $9, \quad E P_{i}=100\left(\frac{Q_{i, \text { exp }}-Q_{i, v}}{Q_{i, \text { exp }}}\right) \quad \mathrm{e}$ $E P_{i}=100\left(\frac{Q_{i, \text { exp }}-\hat{Q}_{i, g}}{Q_{i, \text { exp }}}\right)$ são o erro em porcentagem para a $i$-ésima observação $(i=1,2, \ldots, n)$. Esse valor leva em conta um sinal, designando se a função subestima (+) ou superestima $(-)$ o valor observado. A Equação 9 pode ser escrita da mesma forma que a Equação 10 e 11:

$$
\begin{aligned}
& E P A M=\frac{100}{n} \sum_{i=1}^{n}\left|\frac{Q_{i, \exp }-Q_{i, v}}{Q_{i, \exp }}\right| \\
& E P A M=\frac{100}{n} \sum_{i=1}^{n}\left|\frac{Q_{i, \exp }-\hat{Q}_{i, g}}{Q_{i, \exp }}\right|
\end{aligned}
$$

\section{Avaliação experimental das estacas}

\section{Caracterização das estacas}

Foram estudadas 12 estacas de madeira tratada com CCA da espécie Corymbia citriodora, com 12 $\mathrm{m}$ de comprimento e diâmetro médio de $35 \mathrm{~cm}$, pertencentes à fundação de uma ponte Classe TB45, com dimensões iguais a $10 \mathrm{~m}$ de largura e $12 \mathrm{~m}$ de comprimento, construída em sistema estrutural multicelular de madeira protendida localizada no Campus II da Escola de Engenharia da Universidade de São Paulo, São Carlos, SP, Brasil, conforme a Figura 1.

Em cada lado da ponte foram cravadas 6 estacas, espaçadas em 1,90 m. Do topo de cada estaca foi extraída uma amostra para caracterização, realizada de acordo com os procedimentos da NBR 7190 (ABNT, 1997). Os resultados médios e os coeficientes de variação obtidos foram:

(a) resistência à compressão paralela às fibras: $\mathrm{f}_{\mathrm{c} 0, \mathrm{~m}}=66 \mathrm{MPa}(\mathrm{CV}=18 \%)$;

(b) módulo de elasticidade na compressão paralela às fibras: $\mathrm{E}_{\mathrm{c} 0, \mathrm{~m}}=22.233 \mathrm{MPa}(\mathrm{CV}=$ $34 \%)$; e

(c) peso específico: $9,6 \mathrm{kN} / \mathrm{m}^{3}(\mathrm{CV}=7,5 \%)$.

\section{Caracterização do solo}

Para a caracterização do solo de suporte das estacas foram realizados ensaios Standard Penetration Test (SPT) de acordo com a NBR 6484 (ABNT, 1980) - Execução de sondagens de simples reconhecimento dos solos. Um furo de sondagem foi realizado no local previsto para a cravação de cada uma das estacas, totalizando 12 furos. Assim, foram realizadas 6 sondagens, alinhadas e com espaçamento de 1,90 m de centro a centro, em cada lado da ponte. Nos casos em que a sondagem indicou um valor de $\mathrm{N}_{\mathrm{SPT}}$ diferente da penetração padrão de $30 \mathrm{~cm}$, o valor do $\mathrm{N}_{\mathrm{SPT}}$ correspondente a $30 \mathrm{~cm}$ foi determinado considerando-se uma relação linear entre o valor do $\mathrm{N}_{\mathrm{SPT}}$ e o comprimento de penetração. Todas as propriedades do solo foram determinadas a partir de correlações apresentadas na literatura entre as propriedades necessárias e os ensaios de SPT realizados.

As características médias das camadas de solo do lado da ponte referentes às sondagens SP (1 a 6) e SP (7 a 12) são apresentadas na Tabela 1 .

As características médias do solo de um lado e do outro da ponte não são muito diferentes. De um modo geral, o subsolo pode ser considerado como arenossiltoso medianamente compacto.

Os valores médios de $\mathrm{N}_{\text {SPT }}$ e o coeficiente de variação $(\mathrm{CV})$ para cada metro de profundidade, ao longo das linhas de sondagens SP (1 a 6) e SP (7 a 12), são apresentados na Tabela 2. 
Figura 1 - Vista da ponte multicelular de madeira protendida

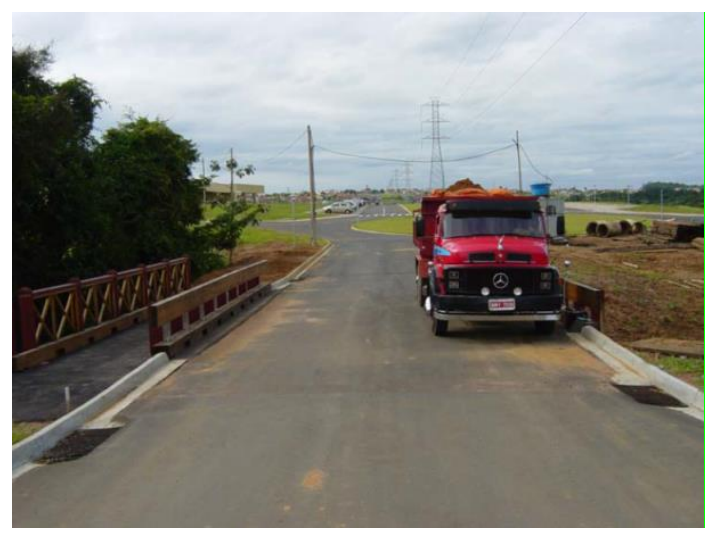

Tabela 1 - Valores das características médias das camadas do solo

\begin{tabular}{|c|c|c|c|}
\hline SP & Prof. (m) & Espessura (m) & Descrição \\
\hline \multirow{4}{*}{1 a 6} & 0,17 & 0,17 & Areia fina pouco argilosa: $\mathrm{N}_{\mathrm{SPT}, \mathrm{med}}=3$ \\
\hline & 2,93 & 2,77 & Areia fina pouco siltosa: $\mathrm{N}_{\mathrm{SPT}, \mathrm{med}}=12$ \\
\hline & 8,12 & 5,35 & Areia fina a média siltosa: $\mathrm{N}_{\mathrm{SPT} \text {,med }}=37$ \\
\hline & 8,12 & $*$ & Impenetrável a ferramenta de percussão: $\mathrm{N}_{\mathrm{SPT} \text {,med }}=47$ \\
\hline \multirow{4}{*}{7 a 12} & 095 & 0,95 & Areia fina pouco argilosa: $\mathrm{N}_{\mathrm{SPT}, \mathrm{med}}=3$ \\
\hline & 3,23 & 2,28 & Areia fina pouco a medianamente siltosa: $\mathrm{N}_{\mathrm{SPT} \text {,med }}=18$ \\
\hline & 7,28 & 4,05 & Areia fina a média siltosa: $\mathrm{N}_{\mathrm{SPT}, \mathrm{med}}=39$ \\
\hline & 7,28 & $*$ & Impenetrável a ferramenta de percussão: $\mathrm{N}_{\mathrm{SPT}, \mathrm{med}}=52$ \\
\hline
\end{tabular}

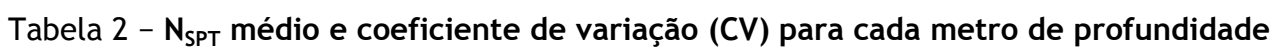

\begin{tabular}{c|c|c|c|c|c}
\hline \multicolumn{3}{c|}{ SP (1 a 6) } & \multicolumn{3}{c}{ SP (7 a 12) } \\
\hline Prof. (m) & N $_{\text {SPT,med }}$ & CV (\%) & Prof. (m) & N $_{\text {SPT,med }}$ & CV (\%) \\
\hline 1 & 6 & 54 & 1 & 3 & 65 \\
2 & 10 & 32 & 2 & 11 & 41 \\
3 & 14 & 23 & 3 & 14 & 17 \\
4 & 17 & 33 & 4 & 29 & 82 \\
5 & 26 & 24 & 5 & 29 & 32 \\
6 & 33 & 23 & 6 & 38 & 39 \\
7 & 36 & 44 & 7 & 50 & 32 \\
8 & 45 & 31 & 8 & 43 & 8 \\
9 & 45 & $*$ & 9 & $*$ & $*$ \\
\hline
\end{tabular}

Os valores médios de $\mathrm{N}_{\mathrm{SPT}}$ para cada metro de profundidade nos dois lados da ponte são semelhantes, e o perfil referente às sondagens SP07 a SP12 apresenta maior variabilidade $(\mathrm{CV}=$ $65 \%$ para $1 \mathrm{~m} \mathrm{e} \mathrm{CV}=82 \%$ para $4 \mathrm{~m}$ ) que o perfil referente às sondagens SP01 a SP06 $(\mathrm{CV}=54 \%$ para $1 \mathrm{~m} \mathrm{e} \mathrm{CV}=44 \%$ para $4 \mathrm{~m}$ ). Os valores de coeficiente de variação evidenciam a grande variabilidade do subsolo quanto à resistência à penetração.

\section{Cravação das estacas}

Foi utilizado bate-estacas com torre de $13,5 \mathrm{~m}$ de altura e mecanismo de movimentação feito por deslizamento sob tubos de aço, apoiados sobre peças de madeira. $\mathrm{O}$ capacete usado tinha $45 \mathrm{~cm}$ de diâmetro e $78 \mathrm{~cm}$ de altura, e foi dividido ao meio por um separador de $3,5 \mathrm{~cm}$ de espessura. A parte superior do capacete era preenchida por um cepo de madeira dura e entre a cabeça da estaca e a parte inferior do capacete não foi utilizado coxim, mas sobre o cepo havia uma chapa de compensado com espessura aproximada de $1,5 \mathrm{~cm}$. $O$ peso total do capacete foi de $1.555 \mathrm{~N}$. As estacas pesavam 10 $\mathrm{kN}$, aproximadamente, e foram cravadas com um martelo de queda livre com $30 \mathrm{kN}$ de peso. A Figura 2 apresenta o bate-estacas com o martelo e o detalhe do capacete utilizado.

O controle da capacidade de carga das estacas de madeira foi feito durante a cravação a partir da medição da nega e do repique, ou seja, por meio do procedimento apresentado por Chellis (1961), 
buscando-se atingir uma carga mobilizada (RMX) maior ou igual ao dobro da carga de serviço de 265 $\mathrm{kN}$, estipulada no projeto estrutural da superestrutura da ponte. Por meio do controle efetuado durante a cravação das estacas, usando-se o método do repique, a relação entre a capacidade de carga avaliada para cada estaca e a carga de trabalho estipulada $(265 \mathrm{kN})$ variou de 2,3 a 4,8 , acima do coeficiente de segurança 2, requerido pela NBR 6122 (ABNT, 2010) - Projeto e execução de fundações. A partir da avaliação experimental durante a cravação das estacas de madeira, recomenda-se utilizar martelo com peso, no mínimo, três vezes maior que o da estaca e altura de queda mínima de $1 \mathrm{~m}$, para cravação de estacas de madeira.

\section{Ensaio de carregamento dinâmico (ECD)}

Foi realizado ensaio de carregamento dinâmico (ECD) em cada uma das 12 estacas que compõem a fundação da ponte, em conformidade com a NBR 13208 (ABNT, 2007) - Estaca: Ensaio de carregamento dinâmico. O equipamento usado para a monitoração das cravações foi um Pile Driving Analyser (PDA), modelo PAK, com seis canais para ligação de instrumentos (transdutores de deformação específica e acelerômetros).

Os instrumentos foram fixados nas estacas aos pares e em posições diametralmente opostas, por meio de parafusos, a uma distância aproximada de três diâmetros da cabeça de cada estaca. Na parte externa das estacas foram instalados dois transdutores de deformação e dois acelerômetros, conforme o procedimento convencional de instrumentação para estacas (Figura 3). Além disso, nesse caso específico de estacas de madeira, instalou-se mais um par de transdutores de deformação específica no interior de cavidades feitas, previamente, nas estacas. Essas cavidades foram feitas em posições diametralmente opostas, com $15 \mathrm{~cm}$ de altura por $10 \mathrm{~cm}$ de largura, e profundidade aproximada de $10 \mathrm{~cm}$. Esse procedimento, inédito, teve a finalidade de possibilitar a determinação de possível diferença entre os módulos de elasticidade dinâmicos das regiões externa e interna da seção transversal das estacas.

O ensaio consistiu na aplicação de uma série de, no mínimo, sete golpes do martelo na estaca, com energias crescentes, isto é, iniciando-se com altura de queda de $20 \mathrm{~cm}$ para o primeiro golpe e acrescentando $20 \mathrm{~cm}$ para os próximos golpes, até $140 \mathrm{~cm}$ para o último. Tal procedimento de execução é denominado ECD de energia crescente ou prova de carga dinâmica. As curvas cargarecalque obtidas por meio desse método experimental, que correspondem à resistência mobilizada versus o deslocamento dinâmico devido ao impacto de energia crescente em cada estaca de madeira estão incluídas na Figura 5.

Figura 2 - Bate-estacas com martelo e capacete
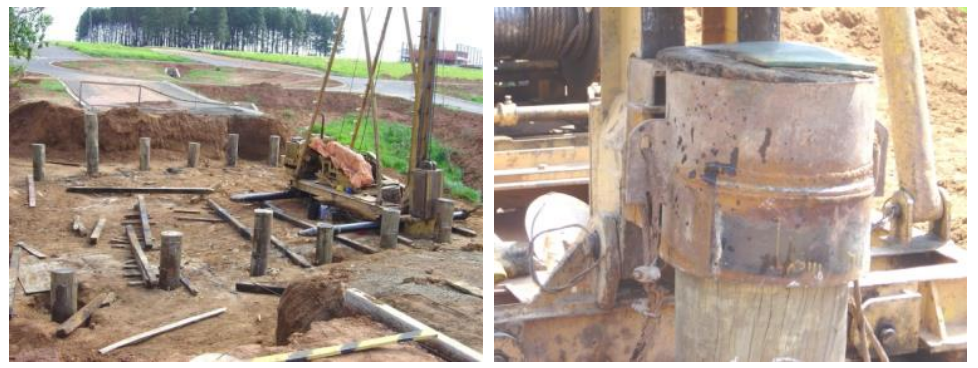

Figura 3 - Instrumentos fixados nas regiões externa e interna da estaca de madeira

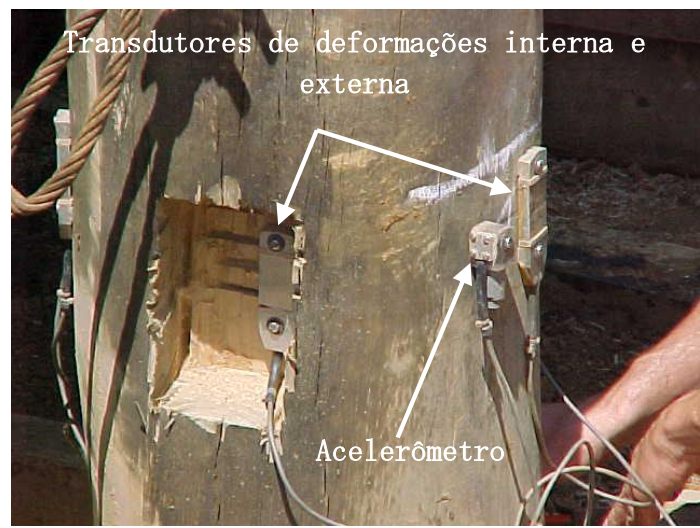

34 Segundinho, P. G. de A.; Miná, A. J. S.; Dias, A. A.; Regazzi, A. J.; Carreira, M. R. 


\section{Resultados e discussão}

Na Tabela 3 são apresentadas as equações estimadas da curva carga-recalque para cada estaca de madeira segundo modelo teórico proposto por Van Der Veen (1953).

Os recalques totais $\left(W^{*}\right)$ para cada estaca, apresentados na Tabela 3, foram definidos a partir do ponto correspondente a uma carga axial igual a $250 \mathrm{kN}$, de acordo com Chellis (1961).

$\mathrm{O}$ valor do coeficiente $\delta_{a}$ foi determinado pela Equação 1, a partir dos valores da capacidade de carga, da carga de trabalho e de seu recalque correspondente. Considerou-se uma distribuição uniforme ao longo de cada trecho da estaca e os parâmetros elásticos do solo, módulo de elasticidade e coeficiente de Poisson, que foram determinados a partir das correlações apresentadas por Teixeira e Godoy (1996).

As equações ajustadas pelo método de Gauss Newton modificado, para cada estaca de madeira, tendo como variável dependente a carga $\left(Q_{i, g}\right)$ e variável independente $\mathrm{o}$ recalque $\left(W_{i}\right)$, são apresentadas na Tabela 4 , bem como os coeficientes de determinação ajustados $\left(R_{a j .}^{2}\right)$ e o intervalo de confiança para os parâmetros $Q_{u, g}$ e $\delta_{g}$. Os valores iniciais dos parâmetros foram a capacidade de carga $\left(Q_{u, a}\right)$, obtida conforme Aoki e Velloso (1975), e o coeficiente $\left(\delta_{a}\right)$ utilizado nas curvas carga-recalque, obtido conforme Van Der Veen (1953).

Das doze equações ajustadas, o menor valor obtido para o coeficiente de determinação ajustado foi de $94,3 \%$.

Na Tabela 5 são apresentadas as capacidades de carga $\left(Q_{u, a}\right),\left(Q_{u, g}\right)$ e $\left(Q_{u, \exp }\right)$ determinadas por meio do método Aoki e Velloso (1975), equações de regressão não linear, ajustadas por meio do método de Gauss Newton modificado e do método Case Pile Wave Analysis Program (CAPWAP) (SMITH, 1960), realizadas nos dados obtidos nos ensaios de carregamentos dinâmicos, respectivamente.

A partir dos dados da Tabela 5, e considerando dados emparelhados, constatou-se que na comparação das médias de $Q_{u, a} \times Q_{u, g}, Q_{u, a} \times Q_{u, \text { exp }}$ e $Q_{u, g} \times Q_{u \text { exp }}$, pelo teste $t$ de Student, elas não diferiram estatisticamente $(\mathrm{P}>0,01)$. Nota-se que os valores apresentaram variação relativa na faixa de $25,1 \%$ a $34,4 \%$.

Tabela 3 - Equações para as estacas de madeira, conforme Van Der Veen (1953)

\begin{tabular}{c|c|c|c}
\hline Estacas & $\boldsymbol{W}^{*}(\mathbf{m m})$ & $\left.\delta_{a} \mathbf{( m m}^{-1}\right)$ & Equações \\
\hline 1 & 0,736 & 0,630 & $Q_{i, v}=674\left(1-e^{-0,030 W_{i}}\right)$ \\
2 & 1,010 & 0,349 & $Q_{i, v}=841\left(1-e^{-0,349 W_{i}}\right)$ \\
3 & 0,713 & 0,698 & $Q_{i, v}=638\left(1-e^{-0,098 W_{i}}\right)$ \\
4 & 0,731 & 0,263 & $Q_{i, v}=1431\left(1-e^{-0,263 W_{i}}\right)$ \\
5 & 0,839 & 0,416 & $Q_{i, v}=848\left(1-e^{-0,466 W_{i}}\right)$ \\
6 & 0,749 & 0,463 & $Q_{i, v}=854\left(1-e^{-0,463 W_{i}}\right)$ \\
7 & 0,807 & 0,331 & $Q_{i, v}=1067\left(1-e^{-0,331 W_{i}}\right)$ \\
8 & 0,734 & 0,273 & $Q_{i, v}=1376\left(1-e^{-0,273 W_{i}}\right)$ \\
9 & 0,684 & 0,482 & $Q_{i, v}=890\left(1-e^{-0,482 W_{i}}\right)$ \\
10 & 0,954 & 0,352 & $Q_{i, v}=938\left(1-e^{-0,352 W_{i}}\right)$ \\
11 & 0,849 & 0,366 & $Q_{i, v}=936\left(1-e^{-0,366 W_{i}}\right)$ \\
12 & 0,930 & 0,312 & $Q_{i, v}=994\left(1-e^{-0,322 W_{i}}\right)$ \\
\hline
\end{tabular}


Tabela 4 - Equações ajustadas pelo método de Gauss Newton modificado, coeficiente de determinação ajustado $\left(R_{a j .}^{2}\right)$ e intervalo de confiança para os parâmetros

\begin{tabular}{ccccc}
\hline Estacas & Equações ajustadas & $R_{a j .}^{2}(\%)$ & $I C\left(Q_{u, g}\right)_{95 \%}$ & $I C\left(\delta_{g}\right)_{95 \%^{\prime}}$ \\
\hline 1 & $\hat{Q}_{i, g}=1542,1\left(1-e^{-0,1316 W_{i}}\right)$ & 99,3 & $1.282,9 \leq Q_{u, g} \leq 1801,3$ & $0,0923 \leq \delta_{g} \leq 0,1710$ \\
2 & $\hat{Q}_{i, g}=789,7\left(1-e^{-0,2345 W_{i}}\right)$ & 98,1 & $705,9 \leq Q_{u, g} \leq 873,5$ & $0,1658 \leq \delta_{g} \leq 0,3033$ \\
3 & $\hat{Q}_{i, g}=481,1\left(1-e^{-0,2709 W_{i}}\right)$ & 94,3 & $407,7 \leq Q_{u, g} \leq 554,5$ & $0,1219 \leq \delta_{g} \leq 0,4198$ \\
4 & $\hat{Q}_{i, g}=1288,0\left(1-e^{-0,1906 W_{i}}\right)$ & 99,5 & $1.149,2 \leq Q_{u, g} \leq 1426,7$ & $0,1518 \leq \delta_{g} \leq 0,2402$ \\
5 & $\hat{Q}_{i, g}=745,7\left(1-e^{-0,3677 W_{i}}\right)$ & 98,4 & $681,1 \leq Q_{u, g} \leq 810,4$ & $0,2652 \leq \delta_{g} \leq 0,4691$ \\
6 & $\hat{Q}_{i, g}=994,6\left(1-e^{-0,4505 W_{i}}\right)$ & 98,7 & $900,2 \leq Q_{u, g} \leq 1089,1$ & $0,2979 \leq \delta_{g} \leq 0,6030$ \\
7 & $\hat{Q}_{i, g}=810,3\left(1-e^{-0,2322 W_{i}}\right)$ & 98,8 & $755,0 \leq Q_{u, g} \leq 865,6$ & $0,1780 \leq \delta_{g} \leq 0,2865$ \\
8 & $\hat{Q}_{i, g}=558,6\left(1-e^{-0,2271 W_{i}}\right)$ & 98,1 & $516,6 \leq Q_{u, g} \leq 600,5$ & $0,1577 \leq \delta_{g} \leq 0,2971$ \\
9 & $\hat{Q}_{i, g}=1313,7\left(1-e^{-0,1684 W_{i}}\right)$ & 99,6 & $1.201,8 \leq Q_{u, g} \leq 1425,6$ & $0,1391 \leq \delta_{g} \leq 0,1976$ \\
10 & $\hat{Q}_{i, g}=962,1\left(1-e^{-0,3077 W_{i}}\right)$ & 95,7 & $856,6 \leq Q_{u, g} \leq 1067,8$ & $0,1727 \leq \delta_{g} \leq 0,4427$ \\
11 & $\hat{Q}_{i, g}=760,7\left(1-e^{-0,2209 W_{i}}\right)$ & 98,4 & $670,2 \leq Q_{u, g} \leq 851,2$ & $0,1538 \leq \delta_{g} \leq 0,2881$ \\
12 & $\hat{Q}_{i, g}=804,8\left(1-e^{-0,2487 W_{i}}\right)$ & 96,7 & $612,1 \leq Q_{u, g} \leq 997,4$ & $0,0954 \leq \delta_{g} \leq 0,4019$ \\
\hline
\end{tabular}

Tabela 5 - Valores de resistência lateral, resistência de ponta e capacidade de carga incluindo média, desvio padrão (DP) e coeficiente de variação (CV)

\begin{tabular}{cccc}
\hline Estacas & $Q_{u, a}(\mathbf{k N})$ & $Q_{u, g}(\mathbf{k N})$ & $Q_{u, e x p}(\mathbf{k N})$ \\
\hline 1 & 674 & 1542,1 & 1150 \\
2 & 841 & 789,7 & 730 \\
3 & 638 & 481,1 & 500 \\
4 & 1431 & 1288,0 & 1150 \\
5 & 848 & 745,7 & 730 \\
6 & 854 & 994,6 & 990 \\
7 & 1067 & 810,3 & 800 \\
8 & 1376 & 558,6 & 570 \\
9 & 890 & 1313,7 & 1140 \\
10 & 938 & 962,1 & 980 \\
11 & 936 & 760,7 & 690 \\
12 & 994 & 804,8 & 730 \\
\hline Média & 957,3 & 921,0 & 846,7 \\
DP & 240,6 & 317,1 & 228,2 \\
CV $(\%)$ & 25,1 & 34,4 & 27,0 \\
\hline
\end{tabular}

Na Tabela 6 são apresentados os resultados do Erro Absoluto Médio (EAM) e do Erro Percentual Absoluto Médio (EPAM) a partir da comparação dos dados experimentais com as equações ajustadas pelo método de Gauss Newton modificado, e as equações de Van Der Veen (1953).

A partir dos resultados apresentados na Tabela 6, pode-se observar que os valores dos erros (EAM e EPAM) obtidos a partir das equações ajustadas pelo método de Gauss Newton modificado foram sempre menores do que aqueles obtidos pelo proposto por Van Der Veen (1953). Dessa forma, a equação ajustada via teoria de regressão não linear conduz a uma maior exatidão nas estimativas obtidas. Os resultados de EPAM também são apresentados graficamente na Figura 4.

$\mathrm{Na}$ Figura 5 são apresentadas e comparadas graficamente as curvas carga-recalque obtidas experimentalmente, por meio do procedimento de ajuste não linear de Gauss Newton modificado e pelo método de Van Der Veen (1953), para as 12 estacas de madeira. 
Tabela 6 - Valores de Erro Absoluto Médio (EAM) e Erro Percentual Absoluto Médio (EPAM) para as estacas de madeira, obtidos pelo método de Gauss Newton modificado e pelo proposto por Van Der Veen (1953)

\begin{tabular}{ccccc}
\hline & \multicolumn{2}{c}{ Gauss Newton } & \multicolumn{2}{c}{ Van Der Veen } \\
\hline Estacas & EAM $(\mathbf{k N})$ & EPAM $(\boldsymbol{\%})$ & EAM $(\mathbf{k N})$ & EPAM $(\boldsymbol{\%})$ \\
\hline 1 & 22,6 & 3,4 & 292,0 & 31,0 \\
2 & 24,9 & 5,0 & 121,7 & 23,9 \\
3 & 31,0 & 8,0 & 213,3 & 56,0 \\
4 & 22,6 & 3,1 & 219,8 & 27,6 \\
5 & 22,6 & 3,7 & 108,7 & 19,2 \\
6 & 34,2 & 4,2 & 113,7 & 12,9 \\
7 & 20,4 & 3,1 & 292,3 & 43,9 \\
8 & 18,8 & 3,6 & 775,2 & 156,5 \\
9 & 19,6 & 2,4 & 133,0 & 18,3 \\
10 & 54,1 & 6,5 & 55,0 & 6,6 \\
11 & 23,7 & 5,0 & 251,1 & 49,2 \\
12 & 35,6 & 6,0 & 210,1 & 36,3 \\
\hline Média & 23,2 & 4,0 & 211,7 & 29,3 \\
Desvio Padrão & 10,1 & 1,7 & 187,4 & 39,5 \\
\hline
\end{tabular}

Figura 4 - Comparação do Erro Percentual Absoluto Médio (EPAM) para as estacas de madeira, obtidos pelo método de Gauss Newton modificado e pelo proposto por Van Der Veen (1953)

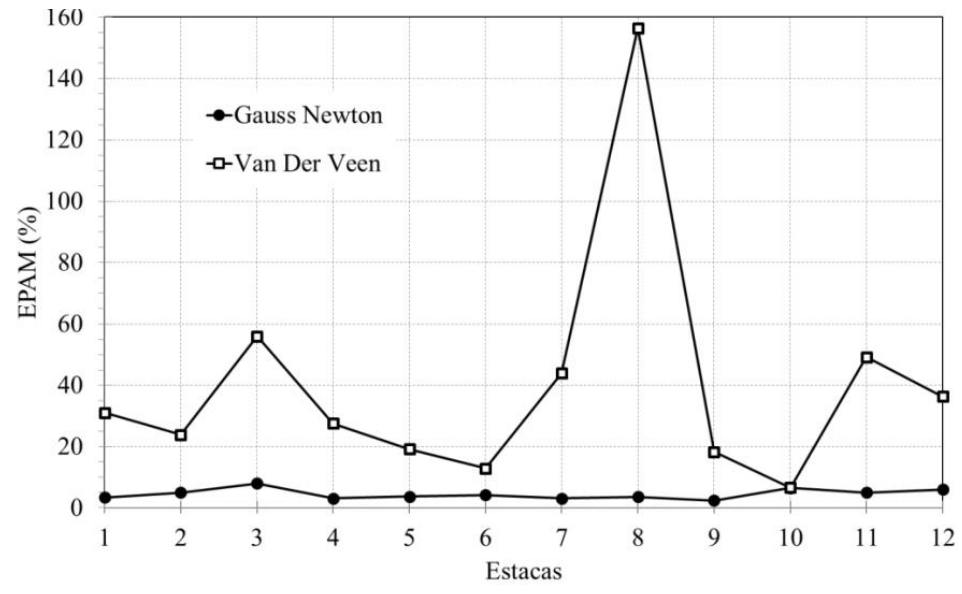

Em geral, os gráficos representados na Figura 5 apresentaram maior concordância entre as curvas carga-recalque obtidas experimentalmente e aquelas ajustadas por meio de regressão não linear em que os parâmetros foram estimados pelo método de Gauss Newton modificado, confirmando os resultados de EAM e EPAM (Tabela 6) e de EPAM (Figura 4). Por outro lado, as curvas carga-recalque obtidas pelo método de Van Der Veen (1953) apresentam concordância distante da experimental, conforme constatado numericamente nos resultados do EAM e EPAM (Tabela 6) e de EPAM (Figura 4).

\section{Conclusões}

A análise de regressão não linear com parâmetros estimados pelo método de Gauss Newton modificado pode ser utilizada para estimar a curva carga-recalque e a capacidade de carga de estacas de madeira a partir de dados experimentais obtidos em ensaios de carregamento dinâmico (ECD). Logo, comprovou-se que esse método não destrutivo, que consiste na aplicação de um carregamento dinâmico axial à estaca, pode ser utilizado em estacas de madeira tomando como base as recomendações de uso expressas na NBR 13208 (ABNT, 2007).

As médias do erro percentual absoluto médio foram $4,0 \%$ e $29,3 \%$, obtidas a partir das equações ajustadas pelo método de Gauss Newton modificado e pelo proposto por Van Der Veen (1953) respectivamente. Portanto, as equações ajustadas via teoria de regressão não linear levaram a uma maior precisão nas estimativas alcançadas, apresentando boa concordância com os dados obtidos experimentalmente. 


\section{Figura 5 - Curvas carga-recalque das estacas de madeira}

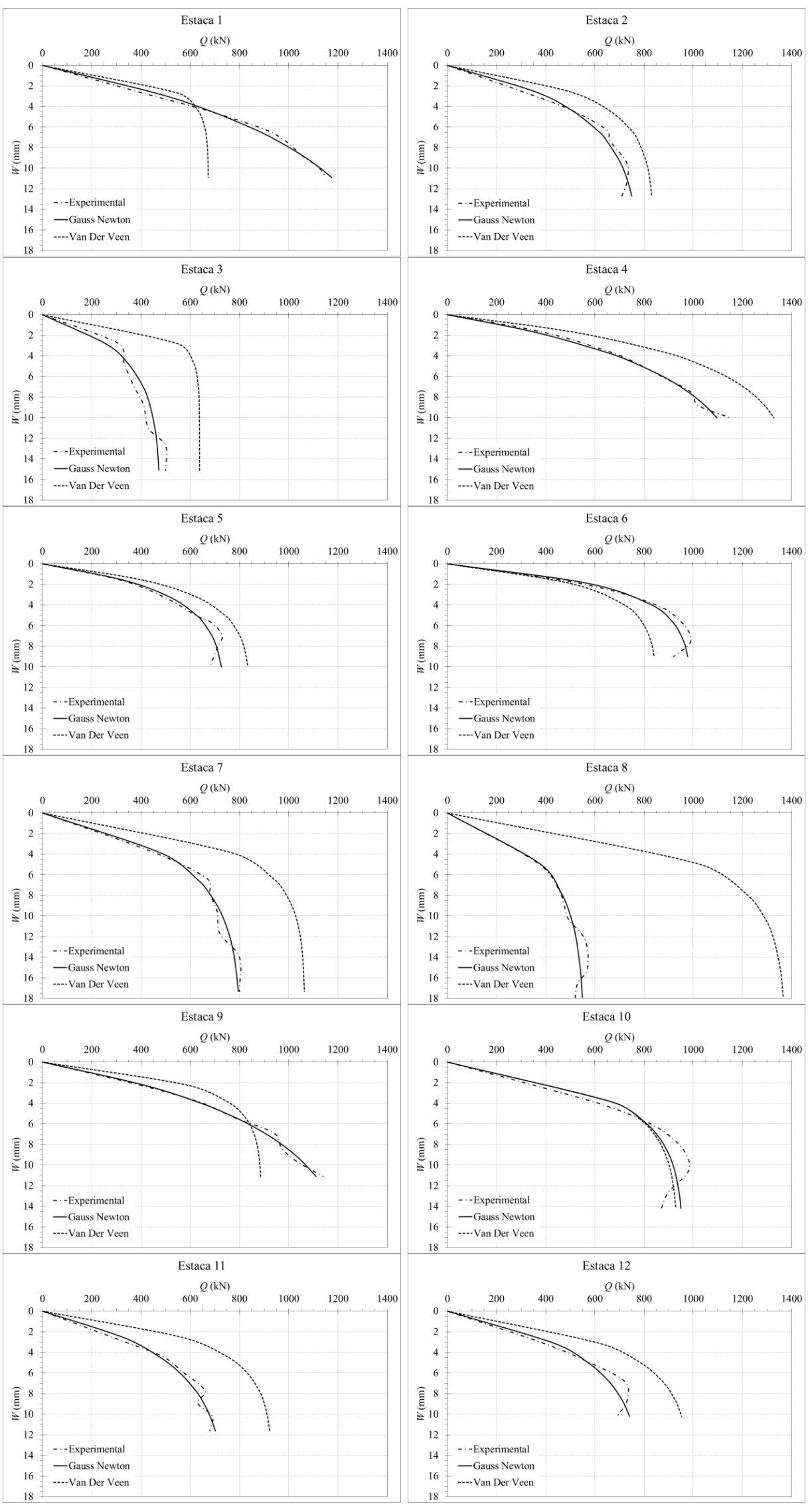

38 Segundinho, P. G. de A.; Miná, A. J. S.; Dias, A. A.; Regazzi, A. J.; Carreira, M. R. 
Recomenda-se, para previsão da capacidade de carga axial de estaca de madeira, usar o método Aoki-Velloso com coeficientes $\mathrm{F}_{1}=3$ e $\mathrm{F}_{2}=4$.

Constatou-se que o uso de estacas de madeira como elemento estrutural de fundação de obras de engenharia, no Brasil, é uma solução ecologicamente sustentável, econômica e estruturalmente viável, que pode ser utilizada para os mais diversos tipos de construções.

\section{Referências}

ALONSO, U. R. Previsão e Controle das Fundações. São Paulo: Edgard Blücher, 1991.

AMERICAN ASSOCIATED OF STATE HIGHWAY OFFICIALS. LRFD Bridge Design Specification. Washington (DC): American Association of State Highway Officials, 2007.

AOKI, N. Aspectos Geotécnicos da Interação Estrutura-Maciço de Solos. In: JORNADAS SULAMERICANAS DE ENGENHARIA ESTRUTURAL, 28., São Carlos, 1997. Anais... São Carlos, 1997. V. 1, p. vii-xx.

AOKI, N.; VELLOSO, D. A. An Approximate Method to Estimate the Bearing Capacity of Piles. In: PANAMERICAN CONFERENCE ON SOIL MECHANICS AND FOUNDATION ENGINEERING (PCSMFE), 5., Buenos Aires, 1975. Proceedings... Buenos Aires, 1975.

ASSOCIAÇÃO BRASILEIRA DE NORMAS TÉCNICAS. NBR 6484: execução de sondagens de simples reconhecimento dos solos. Rio de Janeiro, 1980.

ASSOCIAÇÃO BRASILEIRA DE NORMAS TÉCNICAS. NBR 13208: estacas: ensaios de carregamento dinâmico. Rio de Janeiro, 2007.

ASSOCIAÇÃO BRASILEIRA DE NORMAS TÉCNICAS. NBR 6122: projeto e execução de fundações. Rio de Janeiro, 2010.

ASSOCIAÇÃO BRASILEIRA DE NORMAS TÉCNICAS. NBR 7190: projeto de estruturas de madeira. Rio de Janeiro, 1997.

AMERICAN SOCIETY FOR TESTING AND MATERIALS. D-25 Standard Specification For Round Timber Piles. Philadelphia, PA, 1999.

AMERICAN WOOD PRESERVES INSTITUTE. Timber Pile Design and Construction Manual. Vancouver: Timber Pilling Council, 2002.

BORELLO, D. J. et al. Experimental and Analytical Investigation of Bridge Timber Piles Under Eccentric Loads. Engineering Structures, v. 32, n. 8, p. 2237-2246, 2010.
CHELLIS, R. D. Pile Foundations. $2^{\text {nd }}$. ed. Tokyo: Mc Graw-Hill Book Company, 1961. DÉCOURT, L. Análise e Projeto de Fundações Profundas. In: FUNDAÇÕES: teoria e prática. São Paulo: Pini, 1996.

FREEDMAN, G.; METTEM, C.; LARSEN, P.; EDWARDS, S.; REYNOLDS, T.; ENJILY, V.

Timber Bridges and Foundations: a report produced for the Forestry Commission, Nov. 2002.

KVALSETH, T. O. Cautionary Note About $\mathrm{R}^{2}$. American Statistician, v. 39, n. 4, p. 279-285, 1985.

OKIMOTO, F. S.; CALIL JUNIOR, C. Pontes Protendidas de Madeira: conceitos e aplicações. In: ENCONTRO BRASILEIRO DE MADEIRAS E ESTRUTURAS DE MADEIRA, 6., Florianópolis, 1998. Anais... Florianópolis, 1998. v. 2. p. 303312.

PECK, R. B.; HANSON, W. E.; THORNBURN, T. H. Foundation Engineering. New York: John Wiley \& Sons, 1974.

SMITH, E. A. L. Pile Driving Analysis by the Wave Equation. Journal of Soil Mechanics and Foundations ASCE, v. 86, ago. 1960.

SOUZA, G. S. Introdução aos Modelos de Regressão Linear e Não-Linear. Brasília, DF: Serviço de Produção e Informação, 1998.

TEIXEIRA, A.; GODOY, N. S. Análise, Projeto e Execução de Fundações Rasas. In:

FUNDAÇÕES: teoria e prática. São Paulo: Pini, 1996. p. 227-264.

TSCHEBOTARIOFF, G. P. Fundações, Estruturas de Arrimo e Obras de Terra: a arte de construir e suas bases científicas na mecânica dos solos. São Paulo: Mc Graw-Hill do Brasil, 1978.

VAN DER VEEN, C. The Bearing Capacity of a Pile. In: CONFERENCE ON SOIL MECHANICS FOUNDATION ENGINEERING, 3., Zurich, 1953. Proceedings... Zurich, 1953. v. 2, p. 84-90.

VELLOSO, D. A.; LOPES, F. R. Fundações. Rio de Janeiro: COPPE-UFRJ, 2002. v. 2.

\section{Agradecimentos}

À Fundação de Amparo à Pesquisa do Estado de São Paulo (Fapesp), pelo financiamento de todas as etapas deste trabalho, e à Coordenação de Aperfeiçoamento de Pessoal de Nível Superior (Capes), pela concessão da bolsa. 
Pedro Gutemberg de Alcântara Segundinho

Departamento de Ciências Florestais e da Madeira, Centro de Ciências Agrárias | Universidade Federal do Espírito Santo | Av. Gov. Lindemberg, 316, Centro | Jerônimo Monteiro - ES - Brasil | CEP 29550-000 | Tel.: (28) 3558-2515 |

E-mail: p_gutemberg2001@yahoo.com.br

\section{Alexandre José Soares Miná}

Departamento de Gestão e Tecnologia Agroindustrial, Centro de Ciências Humanas, Sociais e Agrárias | Universidade Federal da Paraíba | Campus Universitário III | Bananeiras - PB - Brasil | CEP 58220-000 | Tel.: (83) 3367-1200 | E-mail: alexminah.uab@gmail.com

\section{Antonio Alves Dias}

Departamento de Engenharia de Estruturas, Escola de Engenharia de São Carlos | Universidade de São Paulo | Av. Trabalhador Sãocarlense, 400, Parque Arnold Schimidt | São Carlos - SP - Brasil | CEP 13566-590 | Tel.: (16) 3373-8207 | E-mail: dias@sc.usp.br

\section{Adair José Regazzi}

Departamento de Estatística | Universidade Federal de Viçosa | Av. P. H. Rolfs s/n , Campus Universitário | Viçosa - MG - Brasil | CEP 36570-000 | Tel.: (31) 9617-1458 | E-mail: adairreg@ufv.br

\section{Marcelo Rodrigo Carreira}

Departamento Acadêmico de Construção Civil | Universidade Tecnológica Federal do Paraná | Via Marginal Rosalina Maria dos Santos | Campus Campo Mourão, Área Urbanizada I | Campo Mourão - PR - Brasil | Caixa Postal 271 | CEP 87301-899 | Tel.: (44) 3518-1400 |

E-mail: carreira@utfpr.edu.br

\section{Revista Ambiente Construído}

Associação Nacional de Tecnologia do Ambiente Construído

Av. Osvaldo Aranha, $99-3^{\circ}$ andar, Centro

Porto Alegre - RS - Brasil

CEP 90035-190

Telefone: +55 (51) 3308-4084

Fax: +55 (51) 3308-4054

www.seer.ufrgs.br/ambienteconstruido

E-mail: ambienteconstruido@ufrgs.br

40 Segundinho, P. G. de A.; Miná, A. J. S.; Dias, A. A.; Regazzi, A. J.; Carreira, M. R. 